\title{
Vergiss mein nicht!
}

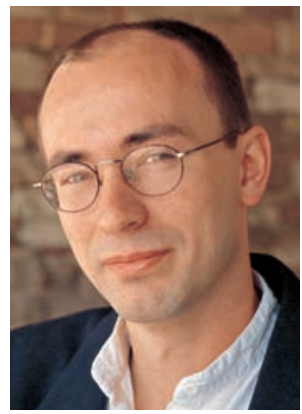

Dr. med. Dieter Schmid, Redaktionsleitung
Da staunten die Münsteraner Ärzte nicht schlecht: Der Patient zeigte ihnen schwer zuzuordnende Flecken auf der Haut, die sich merkwürdig taub anfühlten. Was hatte der bloß? Als die Infektionsexperten der Uniklinik den Fall aufklärten, war die Sensation perfekt: Der Patient litt an Lepra! Offensichtlich hatte er sich bei einem Lateinamerika-Aufenthalt angesteckt. Für eine deutsche Klinik ist ein solcher Patient fast so spektakulär wie die Landung eines Aliens. Weltweit gesehen ist die Situation etwas anders: Pro Jahr erkranken immer noch eine Viertelmillion Menschen an Lepra. Dieses Beispiel zeigt: Unsere hiesige medizinische Versorgung mag noch so umfassend sein - sie deckt nur einen Ausschnitt von dem ab, was das Gros der Menschen weltweit bedroht. Allein schon die 17 von der WHO aufgelisteten „neglected diseases“ wie Lepra, Schlafkrankheit und Trachom quälen weltweit eine Milliarde Menschen. Noch viel mehr sind von den Leiden betroffen, die durch Hygienedefizite, Kriege, Überbevölkerung und Hunger ausgelöst werden. Eine Wissenschaft, die diese Probleme aus der „Vogelperspektive“ betrachtet und Konzepte entwickelt, mit denen die Gesundheit der Menschheit insgesamt verbessert werden kann, ist das Fach „Public Health“. Die AG Public Health der bvmd bietet Projekte an, bei denen Studenten ihren Blick auf die globale Dimension von Gesundheit weiten können. Egal, ob Sie Menschen in Burkina Faso über Malaria aufklären oder eine „Community-Health-Analyse“ in Kenia durchführen: Hier lernen Sie Aspekte der Medizin kennen, die in Deutschland oft vergessen werden. Mehr über dieses Angebot erfahren Sie im Artikel „Global denken, lokal helfen“ auf S. 8.

Natürlich gibt es auch hierzulande Missstände, die von der Öffentlichkeit gerne ausgeblendet werden. Ein Problem rückt derzeit angesichts der Flüchtlingsströme aus Nordafrika wieder in den Vordergrund: die Gesundheit „illegal“ bei uns lebender Flüchtlinge. Diese Menschen sind medizinisch extrem schlecht versorgt. Auch in diesem Bereich können sich Medizinstudenten sehr sinnvoll engagieren - z. B. in einer der vielen „Medinetz“-Gruppen. Mehr dazu lesen Sie im Artikel „Patienten dritter Klasse“ auf S. 30. Eine weitere gesundheitsgefährdete „Bevölkerungsgruppe“, die bisher nur sehr schlecht ärztlich betreut wird, sind fanatische Computerspieler. Schätzungen gehen davon aus, dass $9 \%$ der Jugendlichen hierzulande ein Verhältnis zu ihrem PC haben, das der Gier eines Alkoholikers nach der Schnapsflasche ähnelt. Im Artikel „Im Sog der Scheinwelt“ (S. 52) erfahren Sie alles über die Krankheit Computerspielabhängigkeit (CSA), die so jung ist, dass sie noch nicht einmal im Diagnoseverschlüsselungssystem ICD-10 auftaucht.

Läuft Ihr PC gerade? Mein Tipp: Schalten Sie ihn aus. Vergessen Sie alles andere, lehnen Sie sich zurück - und lesen Sie diese Via medici. Herzlichst, Ihr

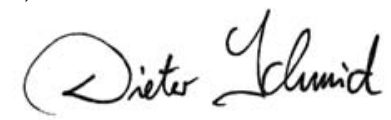

ONLINE-UMFRAGE

\section{Krankenschein für in Deutschland illegal lebende Menschen?}

Hierzulande leben bis zu 1,5 Millionen Menschen ohne Aufenthaltsgenehmigung. Obwohl ihnen nach dem Gesetz medizinische Versorgung zustünde, können sie diese in vielen Fällen praktisch nicht wahrnehmen: Denn der Besuch beim Arzt birgt die Gefahr, entdeckt und abgeschoben zu werden. Aus diesem Grund gehen viele Migranten ohne Papiere oft erst sehr spät zum Arzt und werden dadurch häufig kränker als nötig. Eine Lösung des Problems wäre, einen anonymen Krankenschein für diese Menschen einzuführen. Kritiker der Idee führen an, die Betroffenen seien selbst für ihre Situation verantwortlich. Deswegen könne man für sie wohl kaum die Sozialkassen „einspannen“. Wie stehen Sie zu diesem Thema?

\section{- Sollte die Bundesregierung einen anonymen Krankenschein für Migranten ohne Papiere einführen?}

Stellen Sie sich dieser kniffligen Frage und kommentieren Sie unter: www.thieme.de/viamedici/ aktuelles/aktion/miniumfrage2-11.html
- Ergebnis der Umfrage in Via medici 1.11: Wir fragten, ob die Allgemeinmedizin als berufliche Perspektive für Sie in Frage kommt. Satte 73\% können sich ein Dasein als Hausarzt vorstellen. Für $22 \%$ ist ein Leben mit abendlichen Hausbesuchen dagegen keine Alternative. Einige der Allgemeinmediziner in spe träumten bereits zu Beginn ihres Studiums davon, als Hausarzt ein „intensiveres Verhältnis als in der Klinik zu den Patienten “ aufbauen zu können. Die Gegenseite hält den Beruf aufgrund der aktuellen Bedingungen „für unattraktiv und mit finanziellen Risiken belastet“. Mehr Kommentare unter: .../aktuelles/aktion/miniumfrage1-11.html 